\title{
Smart Home Entertainment System with Personalized Recommendation and Speech Emotion Recognition Support
}

\author{
Zheng Wan \\ School of Information Technology, \\ Jiangxi University of Finance and Economics, \\ Nanchang, China, 330013 \\ wanzheng97@163.com
}

\begin{abstract}
In this paper a personalized recommendation and speech emotion recognition based smart home entertainment system, which integrates multiple entertainment resources including online movies/TV serial, TV programs and online music, is proposed. The system is controlled by the smart phone through finger touch or speech, avoiding searching infrared remote controller. There is no requirement for luxury and expensive acoustic system or smart TV. Furthermore, advertisements are vemoved when watching online movies by capturing video source fites and customizing the web video player. Massive design details are presented and discussed, including: (1) system architecture and functionalities; (2) design of the (server and the smart phone APP; (3) details of personalized recommendation and speech emetion recognition; (4) design of database and some implementation issûts, including theby page source file crawling, regular expression based information extraction, cresource searching and absolute progress locating of web video player.
\end{abstract}

Keywords: Smart home; Entertainment system; Personalized recommendation; Speech Emotion Recognition, Web crawling

\section{Introduction}

With the development of technology and society, higher quality of life is expected, especially in the field of daily living. At home, there are two categories of requirements: one is integrated control of all kinds of devices such as lamps, curtains and domestic appliances [1]. The other is smart service which means that devices can feel the requirements of man and satisfy them automatically [2-4]. Thus, the concept of smart home was proposed $[5,6]$.

Ordinarily, a smart home system consists of several subsystems such as home wiring system, lighting system $[7,8]$, security system $[9,10]$, entertainment system $[11,12]$ and environment control system. Among these subsystems, smart home entertainment system catches researcher's attention because most people spend many hours on entertainment every day including online movies, TV serials and music.

There are three kinds of smart entertainment systems. One is based mainly on acoustic system, such as "Samsung HT-F9730W Smart Home Theater System" and "Sony BDVE3100 Smart Blue-ray Home Theater System". Another is TV based system which runs an android operating system and can be used to watch online movies. The last one is smart home based system, which uses smart phone or tablet PC to control devices. Functionalities of all these systems are predefined so that it is hard for them to feel the requirements of the user.

In this paper we propose a smart home entertainment system with personalized recommendation and speech emotion recognition. The proposed system has the following features: 
(1) No functionality requirements on system acoustic or TV. Only normal smart phone, $\mathrm{PC}$ and TV are needed.

(2) Integrate multiple kinds of entertainment resources: online movies and TV serials, TV programs and online music. Furthermore, updated resource information is provided. Online resources can be searched and played on TV screen with the help of PC.

(3) Remote controller free. All control commands can be issued from the smart phone with input support of finger touch and speech recognition.

(4) No Advertisement. A customized web video player is designed to delete any AD.

(5) Personalized recommendation is support when the user obtains updated resources or searches for specific resources using one or more keywords.

(6) Emotion of the user can be recognized to assist personalized recommendation.

The rest of the paper is organized as follows. First, system overview is introduced in Section 2. Second, details of server design are presented and discussed in Section 3, including components of the server and designs of several core modules. Third, Section 4 shows design of the smart phone APP. Next, Section 5 and 6 deseribe personalized recommendation and speech emotion recognition respectivery. Then, database design and some implementation issues are presented in Section 7, and 8. Finally, Section 9 concludes the paper.

\section{System Overview}

\subsection{System Framework}

Figure 1 presents the framework of proposed smart home entertainment system. There are three kinds of components. The first one is the smart phone, which acts as the terminal for watching and controlling. Two control methods are provided: voice and touch. The next one is the controlled devices: TV and its set-top box and other devices such as CD/DVD player, network player and)projectors. These devices are controlled by the smart phone indirectly. The last one is the server, which is a PC (desktop or laptop) ordinarily. The server acts ascan intermediary between the smart phone and the controlled devices. Notice that projector always acts as a larger screen of the server, and so does TV. Therefore, the server maybe connected to these two devices via an HDMI or a VGA cable. In addition, TV is also the screen of the set-top box when the user watches TV program.

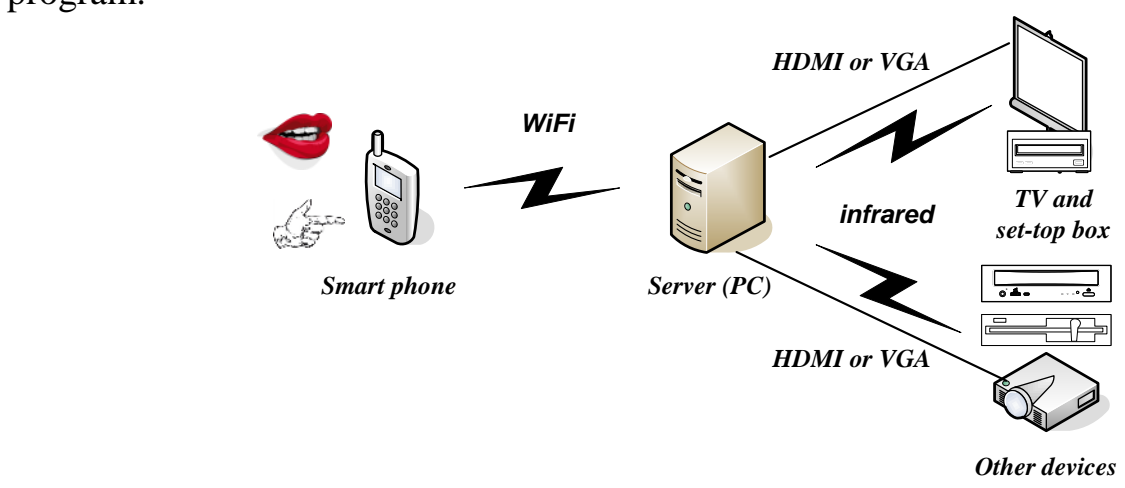

Figure 1. System Framework

The outline of working procedures is as follows:

(1) The user issues a control command within the APP interface of smart phone either by screen touch or by voice. Commonly used commands include "submit a search keyword", "open/close a device", "send a control command while watching", etc. 
(2) Control commands are encoded and sent to the server via Wireless Local Area Network (WLAN, and also denoted as WiFi) between the smart phone and the server.

(3) After receiving and resolving a specific command, the server determines what to do then. Common behaviors include "fetch desired entertainment resources", "play a specific online resource directly", "encode an infrared command and forward it to a specific device", "recognize user's emotion", "recommend entertainment resources", etc.

(4) Controlled devices receive a specific infrared command and take corresponding actions.

\subsection{Functionalities}

Functionalities summary of proposed smart home entertainment system is as follows.

(1) Entertainment resources crawling (including online movies, TV programs and songs ordinarily). The system will fetch the updated entertainment resources automatically and periodically. All these records are stored in the entertaintment pesources database.

(2) Entertainment resources searching. Taking online movjes as an example, the user can find desired online movies by submitting one or more keywords.

(3) Personalized recommendation. According to the interest model of a specific user, a certain amount of possible interested resources (updated resources by crawling or desired resources by searching) are sorted and recommended to the user.

(4) Entertainment resources playing. Continuing with the example of online movies, the user can download, collect or watcha oertain motye in either the recommendation list or the search results immediatefy by issuing a specific command. And during the playing period, controlling conmand such as volume adjustment, fast forward, pause, etc, could be send.

(5) Speech Emotion Recognition. If the command is issued by voice, the emotion recognition module willanalyze the voice and recognize the current emotion of the user. The emotion information is adopted to assist personalized recommendation. That is to say the system wit1 tecommend proper entertainment resources according to both interest model and ea)-time emotion of the user.

\section{Design of the Server}

Components and the design details of the server are described in this section, except for personalized recommendation, speech emotion recognition and database. Details of these two key/components will be further discussed in separated sections.

\subsection{Components of the Server}

Figure 2 presents the components of the server.

(1) Crawler. The crawler fetches the updated online movies, TV programs and music information automatically and periodically. Crawling results are stored in the database and picked up and recommended based on the user's interest model when a user logins.

(2) Resource searcher. The searcher supports online movies, TV programs and music search. Different from the crawler, the searcher works passively and its results depend on the search keyword. Not the search results themselves but the user's subsequent operations on them will be stored in the database. These operations provide implicit feedbacks of the user's interest model.

(3) Online resource player. If the user wants to watch a movie or listen to a song, the player will fetch the source file and play it. It is relatively difficult for playing an online movie because the source file is not a simple link. Two techniques are required. One is called "Online Video Capture", which can resolve the final link of 
source file. The other is the interactive web video player, which can communicate with the other modules to return playback status or accept playback control commands.

(4) Communicator. The communicator is used to exchange messages with the smart phone and other modules. Message format is defined to facilitate packet encapsulation and resolution. Also several codes are defined to distinguish different messages.

(5) Infrared. This module is responsible for issuing infrared control command to those infrared devices such as TV and its set-top box, projector, DVD and CD player, etc. The infrared emitter locates in a serial port board which is already converted to a USB port.

(6) Personalized recommendation. This module maintains the interest models for all the users by capturing implicit feedbacks and recommends entertainment resources to a specific user according to his interest model, when either updated resources information arrives or any search result list is prepared.

(7) Speech Emotion Recognition. This module works in â mode câted "Adaptive and Person Specific. At first emotion models of all users are initiated asing the same speech emotion database. As the user keeps issuing voice commands, he is advised to determine his emotions at the first 10 30 attempts. Every time the user finishes his emotion determination, his speech emotion model will be improved automatically.

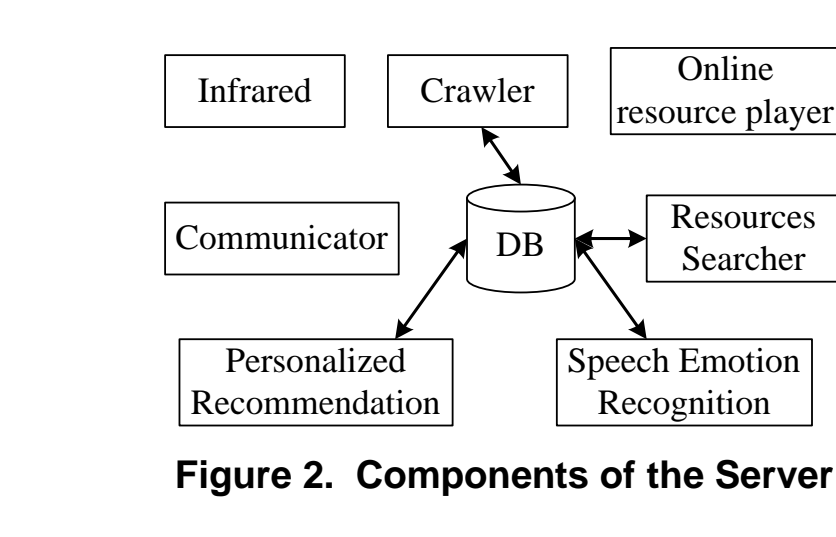

\subsection{Design of the Cravle}

For online movies two sources named Douban and Youku are chosen. The former is a famous website in China which collects the updated movie information and reviews continuously. The latter is a famous online video on demand (VOD) website in China which notony provides videos playing service on its own server but also supports cross database video searching service among several popular VOD websites. TV program schedule is crawled from a website named TV Cat. The schedule is first organized in a txt file and then stored into the database. Music information is crawled from a website named "Baidu Music". Notice that the updated online movies and songs information will be stored permanently in the database while the TV program schedule is stored temporarily because TV programs are real time.

Working process of the crawler is presented in Figure 3, no matter which kind of resource is required. 


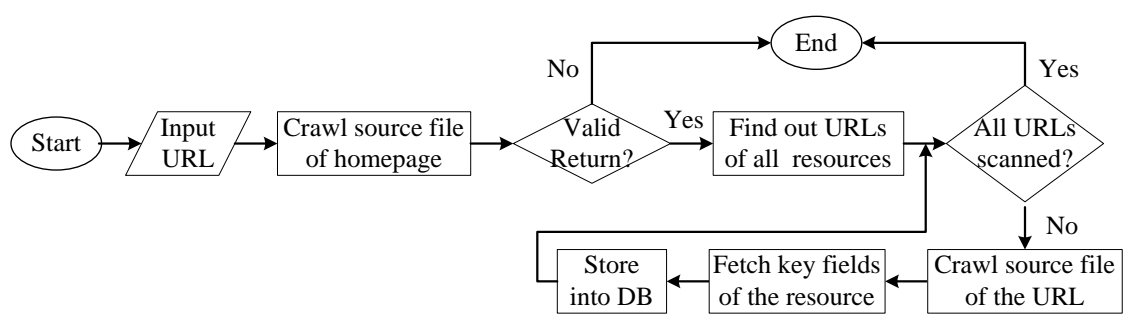

Figure 3. Working Process of the Crawler

(1) Input the URL of the webpage which holds the updated entertainment resources. Table 1 show the URLs used in the proposed system. Actually, these URLs are the homepages of the websites and the updated resource information should be checked further within the pages using proper keyword or sorting criterion. Why not directly employ those URLs which hold updated information? The reason is that thiose URLs are always changed.

Table 1. URLs of Entertainment Resource crawling

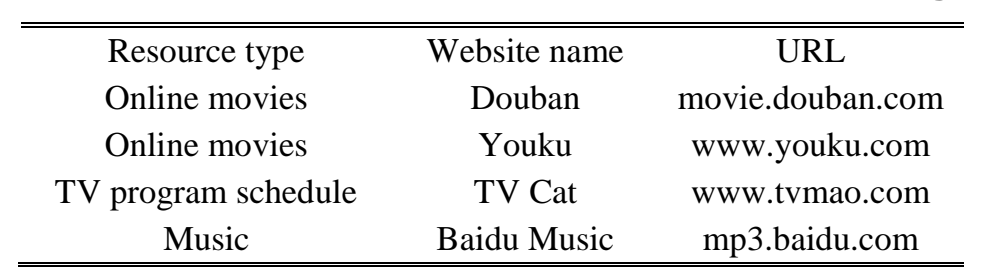

(2) Crawl source file of the updated resoure information pages and judge whether there is a valid return.

(3) If there is no valid return, just jump to the end. Otherwise, search the source file with proper regular expression to find out the URLs of all updated resources.

(4) For each URL of an updated resource, crawl source file of the corresponding webpage.

(5) Search the source file with another proper regular expression to extract data of all those key fields about the updated resource which are needed by the database.

(6) Store data of those fields into the database.

(7) Repeat steps (4) (6) until all URLs are scanned.

A regular expression has two components ordinarily. One is the Pattern, which is the compiled form of the regular expression. The other is the Matcher, which searches the data stream and returns matched results so that the data of those key fields about the updated resource could be fetched.

As an example, the following regular expression is used to fetch the URL of the image whien describes an updated movie. The letters /" actually mean a double quotation mark. All those letters in the regular expression except for $(. *)$ should be matched in the source file. The letter combination (.*) means there can be any number of letters here. If a string matches this regular expression, the letters stand in the position of $\left(.^{*}\right)$ will be fetched.

$$
\text { <img class }=|"| " s r c=\left|"\left({ }^{*}\right)\right| " \text { alt=l"(. *)|"> }
$$

\subsection{Design of Resource Searcher}

Working process of the resource searcher for online movies or music is given in figure 4. The resource searcher for TV programs works differently. Since TV program schedule is achieved in the crawling process, searching will be performed within the temporarily stored schedule. 


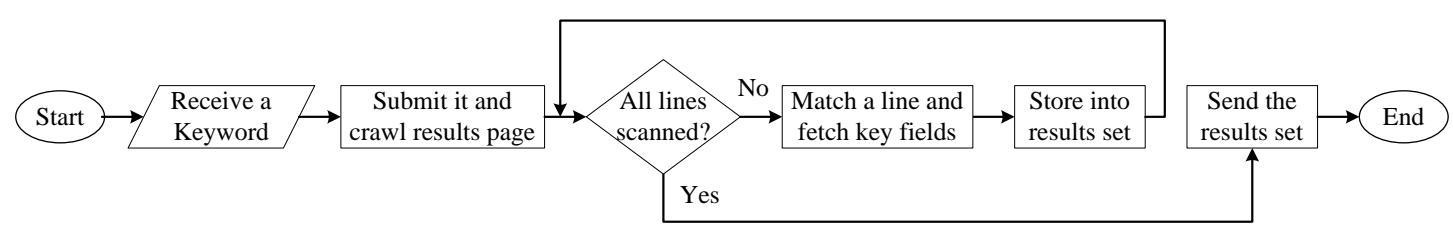

Figure 4. Working Process of Resource Searcher

(1) Receive a search keyword from the smart phone through the communicator.

(2) Take the keyword as parameter and access the results page. Crawl the source file of the results page then. If results page crawling has a valid return, begin to fetch search results.

(3) Match each line with the predefined regular expression and extract data of key fields about the certain result. Then store the new record into the results set.

(4) Repeat step (3) until all lines of the source file of results page are scanned.

\subsection{Design of Online Resource Player}

If the user wants to play a resource, the online resource player will be wake up. In this subsection, we take online movie playing as the example. There are three phases for the player to work.

(1) Achieve the source file of the resource." "OnlineQVideo Capture" technique is employed.

(2) Invoke the web video player.

(3) Receive the control commands from the communicator and forwards them to the player.

3.4.1. Online Video Capture: The conmplete "Online Video Capture" procedures begin with the URL of a resource and end when the source files of the resource are achieved, including lots of HTTP messages exchange. To simplify the procedures, we utilize a capture agent website named Rat. The URL of Rat is www.flycd com. Initial URLs of several divided source files of the video will be returned as long as the URL of the resource is submitted. Then final URLS of the divided source files could be achieved owhen GET requests towards those initial URLs are sent.

Figure 5 shows the working process of online video capture process with the help of Rat.

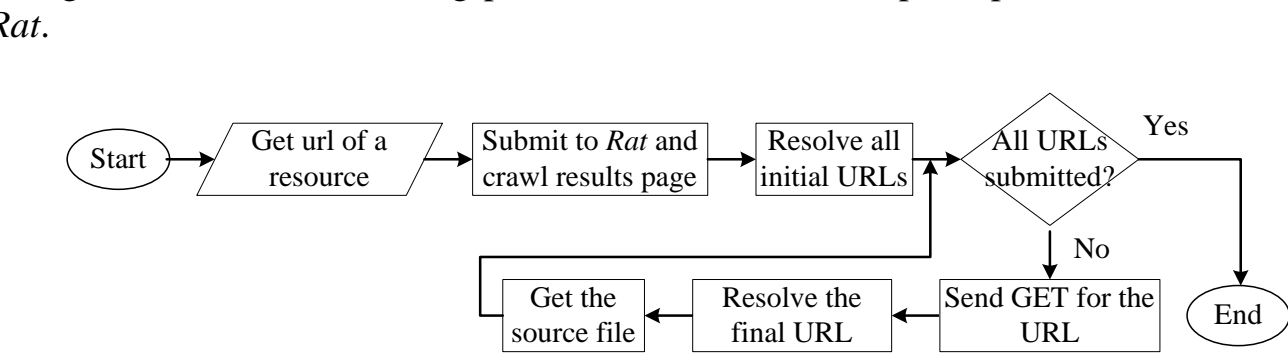

Figure 5. Process of Online Video Capture with the Help of Rat

3.4.2. Web Video Player: The web video player should provide various kinds of API to support operations of video playback, such as URL management, volume control, screen zoom, fast forward/backward, etc. To satisfy the above requirements, the "JW player" is chosen.

JW player is an open source web-based flash video, audio and image player, which supports Sliverlight playing. JW player supports flash and HTML5 videos, including FLV, H.264, MP4 and VP8. It also supports various kinds of streams and playlists, including RTMP and HTTP live. And adaptive bit rate handover is supported too. Furthermore, JW player can be extended based on your own purpose. 
3.4.3. Interactions between Player and other Modules: Most modules are programmed by Java and the web video player is based on JavaScript. To implement interactions between them, the class JWebBrowser is introduced (see Figure 6).

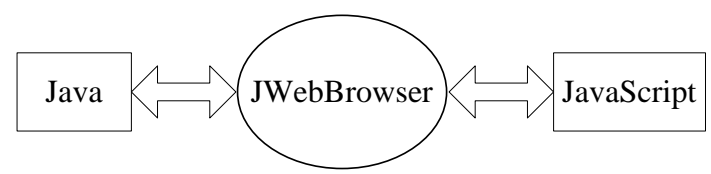

Figure 6. Interactions between Java and JavaScript

In class JWebBrowser, method navigate() is used to load JW player and set basic features. Other operations are performed through method executeJavascript(), which çân pass the JavaScript codes (APIs provided by JW player) to the web page which contains the JW player.

3.4.4. Procedures of Online Video Playback: Figure/7 shows the condplete procedures of online video playback when the video is divided intoreveral segments.

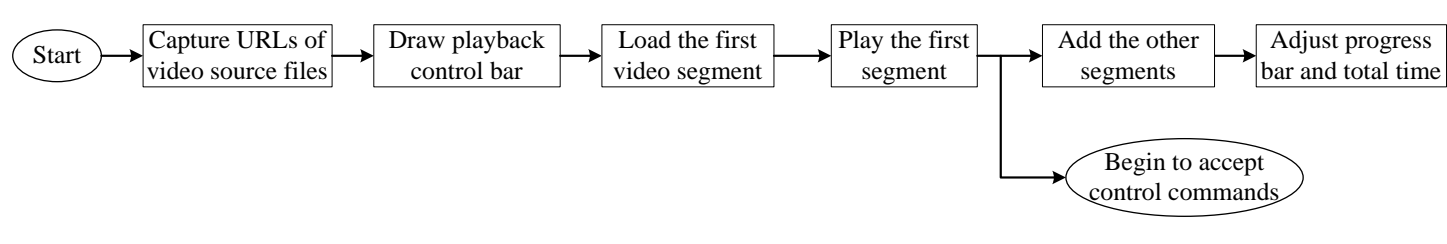

Figure 7. Procedures of Online Video Playback

(1) To improve user experience, a playback control bar is customized which includes functionalities of (play/pause, enter/exit whole screen, fast forward/backward, add/reduce volume mute and absorute progress locating.

(2) Since there are several video segments, only the first segment is loaded at the initialization phase so that less waiting time can be achieved.

(3) After the finst segment begins to play, the player is ready to accept any control command. At the mean time, the other segments are loaded.

(4) When all segments are loaded successfully, the progress bar and total time in the playback contifor bar should be adjusted.

\subsection{Design of Infrared Module}

Infrared module includes several sub-modules and has several kinds of interaction with other module or devices, shown as Figure 8.

(1) Infrared command learning. There is a module to enable infrared device commands learning in the infrared emitter. This module interacts with the controllers of infrared devices such as TV remote controller. When the user presses a button on the controller towards the infrared emitter, the module receives the infrared signal. This module can also interact with the burning program to set mapping codes for different signals.

(2) Infrared emitter driver. The driver makes the emitter usable for the operating system. It is based on cp210x driver, which makes the emitter to be connected by a USB port. Actually, the emitter is a serial device, which works on a baud rate of 9600 . When the server receives a control command from the smart phone, it forwards the command to the driver through java serial communication packages.

(3) Mapping table. Mapping relationships are between infrared signals and button codes of infrared device remote controller are stored in this table. The driver searches the 
table for the receiving code and sends the corresponding infrared signal to the emitter. Finally, the infrared signal could be sent to the infrared devices.

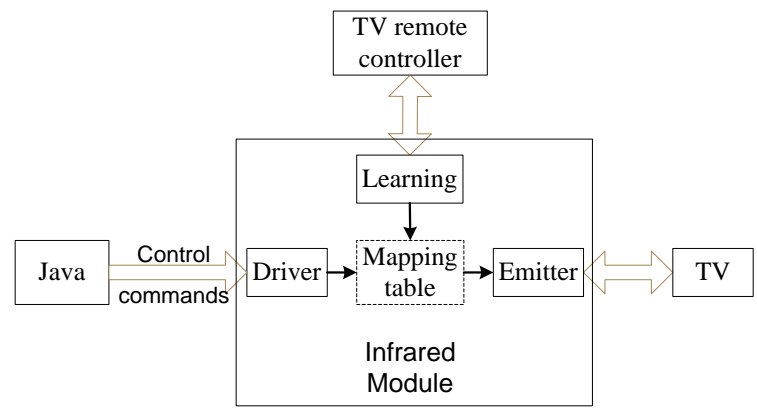

Figure 8. Architecture of Infrared Module

\section{Design of the Smart Phone APP}

Figure 9 describes the framework of the smart phone APP.

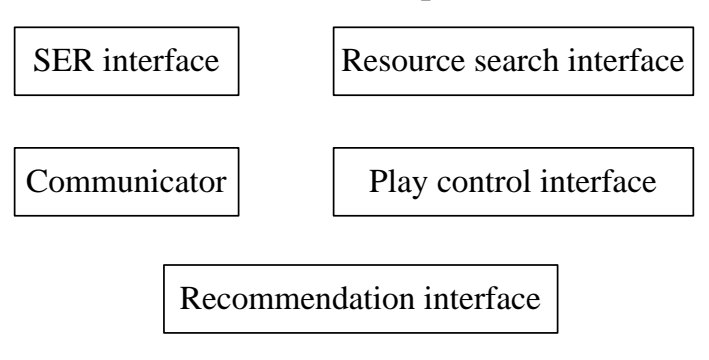

Figure 9. Framework of Smart Phone APP

(1) Resource search interface. This interface accepts the keyword input and transmits it to the communicator. The user first chooses the resource type he wants to search and then input the keywords. Actually, the online resources are divided into two categories further: onlune movies and online TV serials.

(2) Play control interface. This interface receives the operations from the user and transmits them to the communicator.

(3) Communicator. The communicator receives all kinds of information from other modules and the server, and sends encapsulated messages to the server. For example, it receives a control command from the play control interface and encapsulates a corresponding message. Then, the message will be sent through the pre-established socket. The communicator receives search keywords from the resource search inferface, voice from SER interface. And it also receives data from the server such as recommended updated resource information and resource search results.

(4) SER interface. SER is the abbreviation of speech emotion recognition. Actually, this interface is just a speech recognition engine. In proposed system, Google speech recognition engine is employed.

(5) Recommendation interface. The default page of the APP is the updated resource information sorted by date. If the user presses the button "Recommendation", the APP will send a request to the server and list returned recommendations in this interface. 


\section{Personalized Recommendation}

\subsection{User Interest Model}

The user interest model consists of five attribute vectors of a movie: (1) type; (2) district; (3) actor; (4) director; (5) year of production. Each vector contains several attribute value and weight pairs. For example, the following type vector means that there are three pairs in this attribute vector: comedy, action and mystery. That is to say, the user is interest in these three kinds of movies. And the weights of them are 0.35, 0.21 and 0.11 respectively. Thus, the favorite movie type of this user is comedy.

Type vector: $\{($ Comedy, 0.35), (Action, 0.21), (Mystery, 0.11)\}

User interest model can be calculated by the records of ever seen movies. All the keywords of different attributes are counted. Since a keyword's appearance always has its value, the $t f$ equation is adopted to calculate weights. In this equation, $n$ is the times that a keyword appears and $N$ is the total times that all keywords belonging to the same type appear.

$u_{k i}=\frac{n}{n+N}$

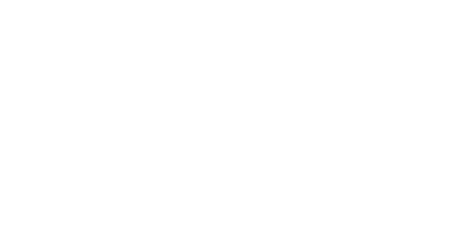

\subsection{Weight Updating}

After the user watched more and more moyres, the interest model should be adjusted, especially for those weights of different attribute values. To update the weights, we introduce two concepts: attribute importance and attribute concentration degree.

(1) Attribute importance reflecs the impact of an attribute on user's choice. Large importance means significant influence. Assume that there are $m$ attributes and the kth attribute has $n$ different values. Then the importance of the $k t h$ attribute can be calculated as:

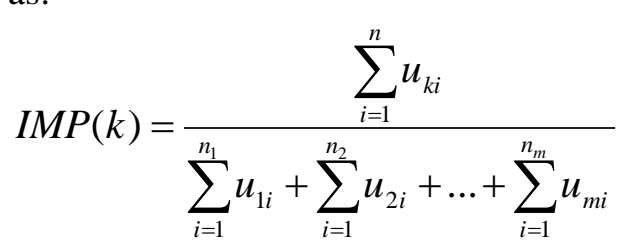

(2) Attribute concentration degree reflects the degree of concentration among different keywords within a speeific attribute. Large degree means more unambiguous interest so that large weight should be set. The equation is as follows $\left(1<=j<=n_{k}-1, j+1<=i<=m_{k}\right)$.

$$
\operatorname{CON}(k)=\sqrt{2 * \sum_{j=1}^{n_{k}-1} \sum_{i=j+1}^{n_{k}}\left(u_{k j}-u_{k i}\right)^{2} / n_{k} *\left(n_{k}-1\right)}
$$

Weight updating equation for a certain weight $w$ is as follows. $N E W(w)$ and $O L D(w)$ mean the newly calculated and the previous value of $w$.

$$
N E W(w)=(\operatorname{IMP}(w)+C O N(w)) \times 0.6+O L D(w) \times 0.4
$$

\subsection{Similarity Calculation}

Since the movie and the user interest model have the same vector format, their similarity can be calculated as follows. 


$$
\operatorname{sim}(f, s)=\cos (f, s)=\frac{\sum_{i=1}^{n} f(i) \times s(i)}{\sum_{i=1}^{n} f^{2}(i) \times \sum_{i=1}^{n} s^{2}(i)}
$$

Similarities of all the five vectors can be calculated by the above equation, add the results altogether to obtain the final similarity. After similarities of all updated movies are calculated, we can sort these movies by their similarities and recommend them to the user.

\section{Speech Emotion Recognition}

Speech Emotion Recognition (SER) module returns the user's current emotion based on his speech control command. Procedures of SER are described in figure 10 and 1

(1) Endpoint detection. Judge the beginning point and ending point of at input speech based on specific speech features so that the valid speech segment could be achieved.

(2) Speech feature extraction. Analyze the valid speech segment to obtain different kinds of speech features such as frequency and amplitude, and stored in corresponding vectors.

(3) Emotion model training. Prepare training samples including speeches and their corresponding emotions and train them by Support Vector Machine (SVM) algorithm to establish the speech emotion model.

(4) Recognition of speech emotion. After the user issues a speech, extract the features within speech and import them to the speech emotion model. The model then returns the recognition result, i.e. which emotion døes the speech reflect?

(5) Adaptive adjustment of speech emotion model. Since the speech emotion models for different persons may be distinct, we add every recognition results to the previous model so that the model caradapt itself to specific person gradually.

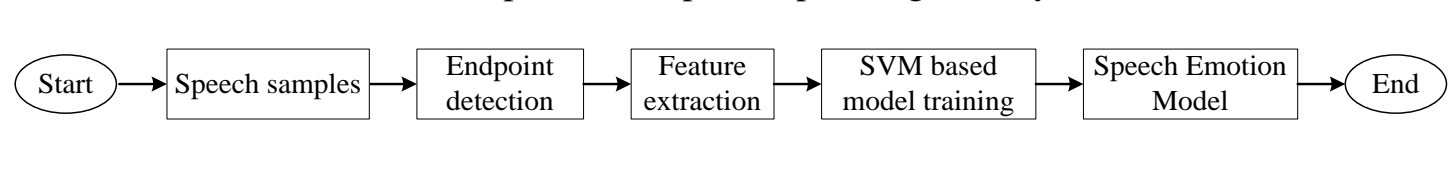

Figure 10. Procedures of Speech Emotion Model Establishment

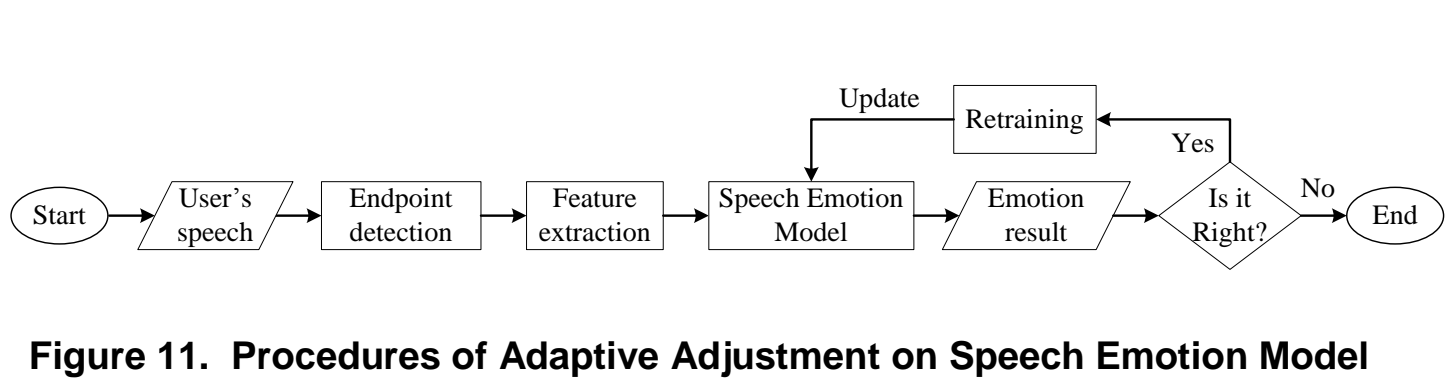

\section{Design of Database}

There are two primary data stored in the database: movie information and user interest model. A movie is identified by its No. named movieNo. Besides, there are many other fields such as actors, directors, district, year of production, etc. Since an actor or a director may take part in different movies, there is no direct relationship between a specific movie and a specific actor or director. Thus we set up a table named Person to hold actors and directors. It consists of two fields: personNo and personName. To distinguish two categories of persons, personNo of an actor begins with a letter $A$ and personNo of a director begins with $D$. Another two tables named Actor and Director establish the 
relationship between persons and movies, which consists of two fields: personNo and movieNo. The other tables about a movie are Type, Area (district of the movie) and Opentime (year of production). Updated movie information is stored in the table named newMoive, which has the same structure as Movie.

User related tables include User (basic information of the user), UserIns (user interest model), UserMovie (ever seen movies of the user) and Recommend (results of personalized recommendation). Data fields of UserIns include userNo, attribute, weight and refreshtime. Data fields of UserMovie include userNo, movieNo, watchTime and watchNum.

Figure 12 describes part of the E-R diagram.

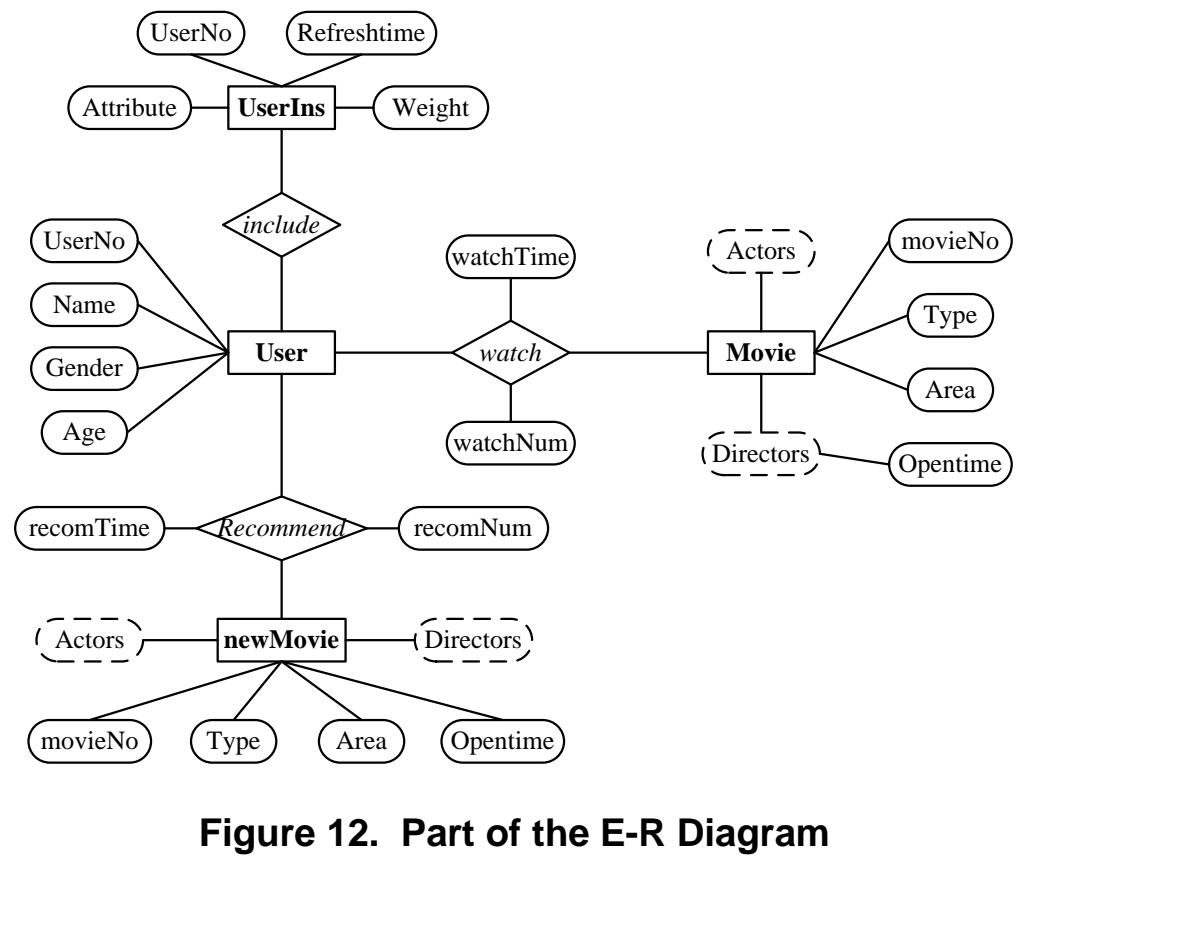

\section{Implementation Issues}

\subsection{Crawl the Source File of a Web Page}

The representation codes of source file crawling are as follows. First, a URL object is created. Then, the URL is accessed and the source codes are returned to a BufferedReader object. Finally, the source codes are written to a TXT file line by line.

URL url = new URL(httpUrl);

BufferedReader ii = new BufferedReader(new InputStreamReader(url.openStream(), "utf-8"));

PrintWriter $p=$ new PrintWriter("douban.txt”);

while $(($ input $=$ ii.readLine ()$) !=$ null) \{ \} p.printIn(input);

\subsection{Regular Expression based Information Extraction}

The codes of information extraction are as follows. First, a regular expression is defined in a Pattern object to find image URL of a movie. Then, the Pattern object is used to match a string $s$ and results are returned and stored in a Matcher object. Finally, valid values of two data fields (imageURL and imageName) are stored. 


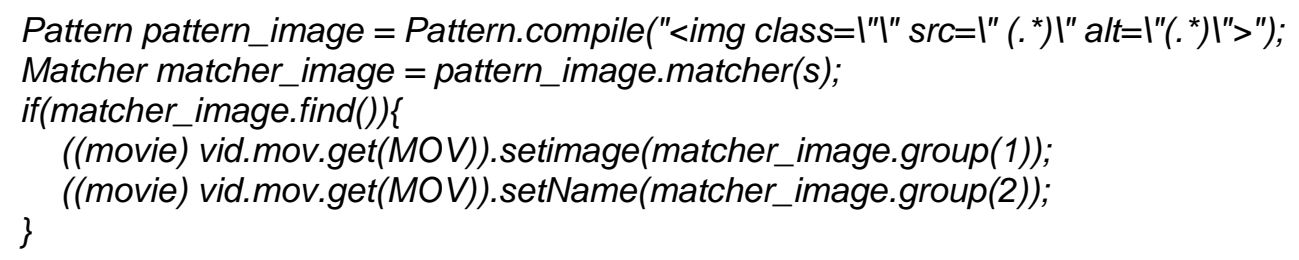

\subsection{Resource Searching}

The pseudo codes of resource searching are as follows. First, the search keyword is obtained from the smart phone APP. Next, the searching URL which takes the keyword as its parameter is established. Then, get the source code of the result page. Finally, extract each result record from the source file.

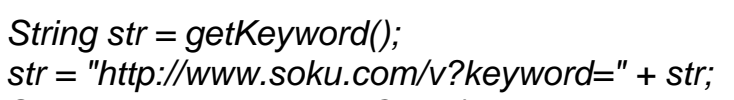

String results = getHtm/Code(str, "searchresults.txt");

Extract result record from searchresults.txt using a regular expression:

\subsection{Absolute Progress Locating of Web Video Player}

The pseudo codes of absolute progress locating are as follows,

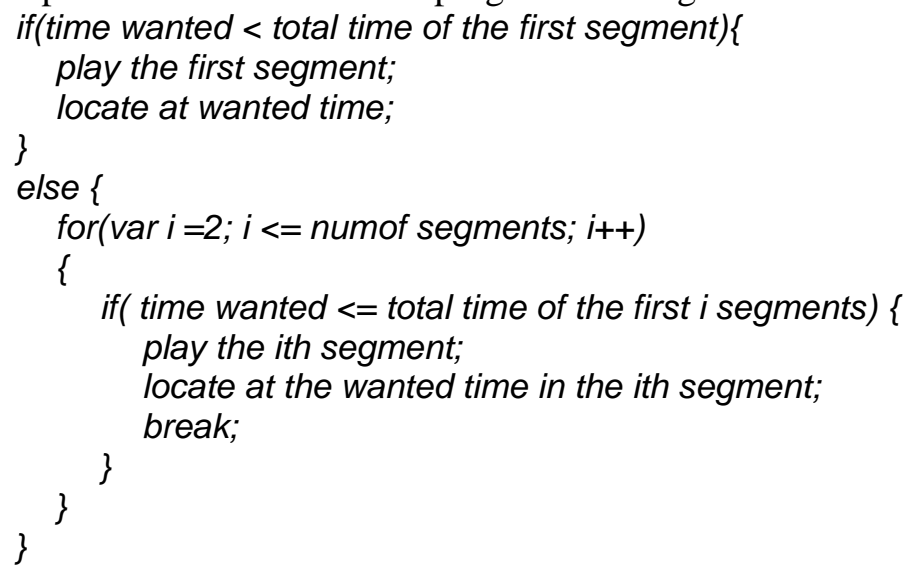

\section{Conclusions}

A personalized recommendation and speech emotion recognition based smart home entertainment system is proposed in this paper, which has five functionalities: (1) entertaimment resources crawling; (2) entertainment resources searching; (3) personalized recommendation; (4) entertainment resources playing; and (5) speech emotion recognition. Characteristics of proposed system include:

(1) No functionality requirements on system acoustic or TV.

(2) Integrate online movies and TV serials, TV programs and online music.

(3) Control commands are issued from the smart phone by finger touch or speech.

(4) No Advertisement when play online videos.

(5) Personalized recommendation for different individuals.

(6) Emotion recognition through speech.

In this paper, many details are presented and discussed: (1) system architecture and functionalities; (2) design of the server (crawler, resource searcher, online resource player and infrared module); (3) design of the smart phone APP; (4) details of speech emotion recognition and personalized recommendation; (5) design of database and (6) some implementation issues, including web page source file crawling, regular expression based 
information extraction, resource searching and absolute progress locating of web video player.

In the future, we plan to design and implement other subsystems of smart home system.

\section{Acknowledgements}

This work was supported by National Natural Science Foundation of China (No. 61162009), Natural Science Foundation of Jiangxi Province (No. 20142BAB217004), Science and Technology Project of Jiangxi Education Department (No. GJJ12273), and Visiting Scholar Special Fund of Young and Middle-Aged Teacher Developing Plan of Jiangxi Ordinary Universities.

\section{References}

[1] Mingfu Li and Hung-Ju Lin. Design and Implementation of Smart Home Control Systerns Based on Wireless Sensor Networks and Power Line Communications. IEEE Transactions on Industrial Electronics, 2015, 62(7): 4430-4442.

[2] Dong-oh Kang, Kyuchang Kang, Jang-Ho Choi and Changseok Bae. A bridge structure of device sociality framework for smart home service. Proceeding of IEEE Líternational Symposium on Consumer Electronics, pp.1-2, (2010) June 22-25; Jeju, South Korea.

[3] JinSung Byun; Boungju Jeon; Junyoung Noh; Youngil Klm and Sehyun Park. An intelligent selfadjusting sensor for smart home services based on ZigBee communications. IEEE Transactions on Consumer Electronics, 2012, 58(3): 794-802.

[4] Zion Hwang; Yoonsik Uhm; Yong Kim; Gwanyeon Kim; SehyumPark. Development of LED smart switch with light-weight middleware for location-aware services in smart home. IEEE Transactions on Consumer Electronics, 2010, 56(3): 1395-(1402.

[5] J. H. Jahnke, M. d'Entremont, J. Stier Facilitating the programming of the smart home. IEEE Wireless Communications, 2002, 9(6): 70- 6 .

[6] K.Wacks. Home systems standards: achievements and challenges. IEEE Communications Magazine, 2002, 40(4): 152-159.

[7] N. Chandrakar, S. Kaul, M.Mohan, C. Sai Vamsi and K. R. Prabhu. NFC based profiling of smart home lighting system. Proceedings of Internationa Conference on Industrial Instrumentation and Control, pp.338-341, (2015) May 28-30; Maharashtra, India.

[8] J. Garcia-Guzman, E. \%. Moctezuma Monge and F. H. Villa-Lopez. FPGA implementation of a smart home lighting control system. Proceedings of IEEE International Conference on Consumer Electronics Berlin, pp.419-420, (2014) September 7-10; Berlin, Germany.

[9] Changmin Lee, L. Zappaterra, Kwanghee Choi and Hyeong-Ah Choi. Securing smart home: Technologies, security challenges, and security requirements. Proceedings of Communications and Network Security, pp.67-72, (2014) October 29-31; San Francisco, USA.

[10] Yuanbo Xu, Yu Jiang, Chengquan Hu, Hui Chen, Lili He and Yinghui Cao. A balanced security protocol of Wireless Sensor Network for Smart Home. Proceedings of International Conference on Signal Processing, pp.2324-2327, (2014) October 19-23; Hangzhou, China.

[11] Shuyan Jiang; Jie Peng; Zhi Lu; Junjie Jiao; Shanshan Jiang. 802.11ad Key Performance Analysis and Its Application in Home Wireless Entertainment. Proceedings of IEEE International Conference on Computational Science and Engineering, pp.1595-1598, (2014) December 19-21; Chengdu, China.

[12] P. N. Borza, M. Romanca, V. Delgado-Gomes. Embedding patient remote monitoring and assistive facilities on home multimedia systems. Proceedings of Optimization of Electrical and Electronic Equipment, pp.873-879, (2014) May 22-24; Brasov, Romania.

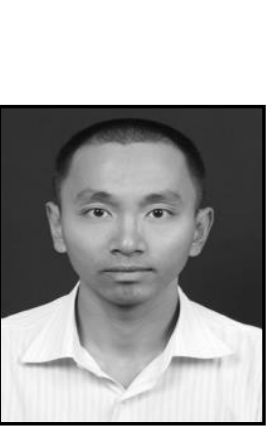

\section{Author}

Zheng Wan, He received the B.E. and Ph.D. degree in computer science in 2001 and 2006 from Zhejiang University of China. He is now an associated professor of Jiangxi University of Finance and Economics, Nanchang, China. He is currently working in the areas of wireless video communications, smart home system, and development of Android system and applications. 
International Journal of Smart Home

Vol. 10, No. 8 (2016)

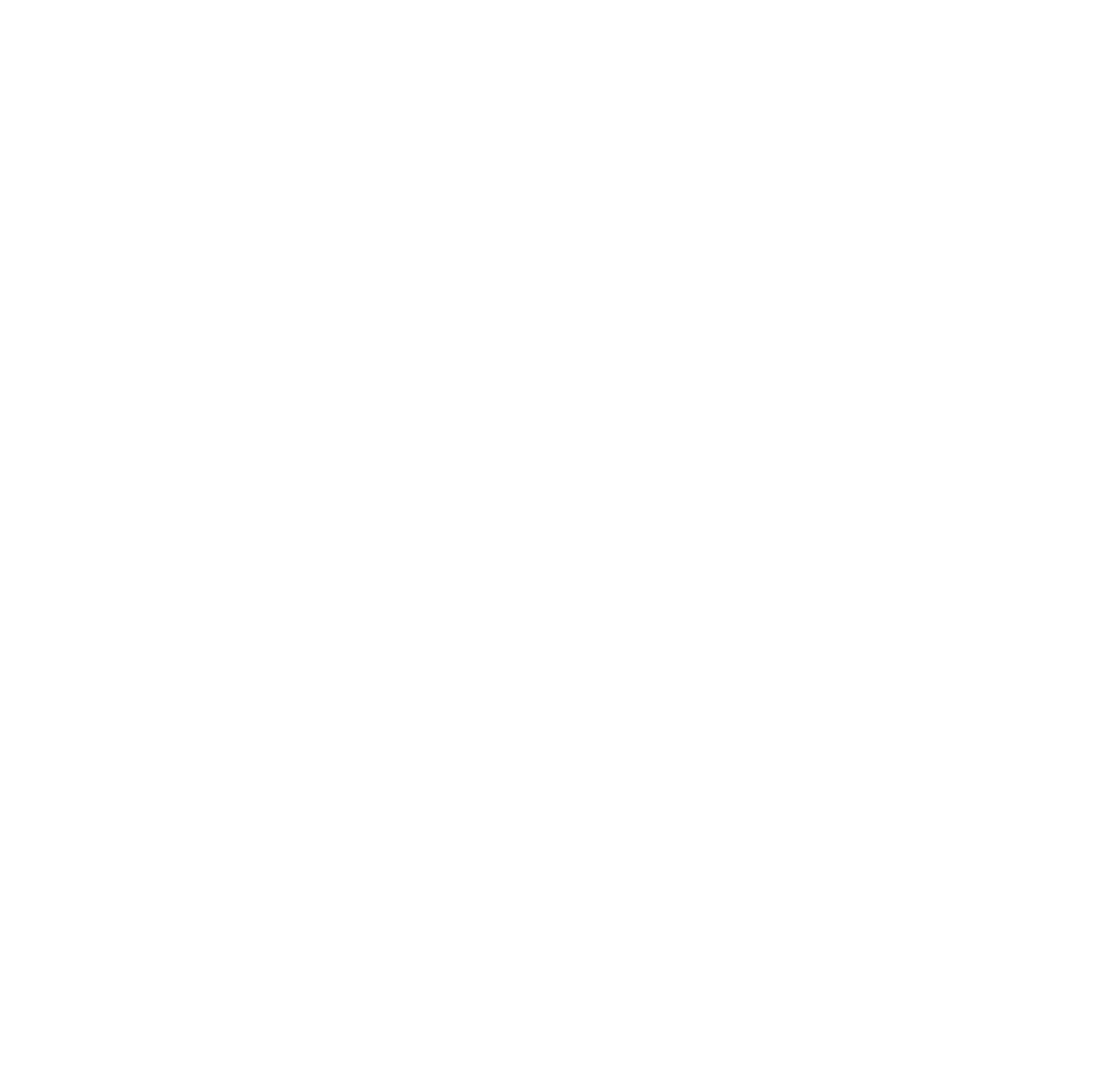

